\title{
SURVEY OF SUPER RESOLUTION TECHNIQUES
}

\author{
Anand Deshpande ${ }^{1}$ and Prashant P. Patavardhan ${ }^{2}$ \\ ${ }^{1}$ Department of Electronics and Communication Engineering, Angadi Institute of Technology and Management, India \\ ${ }^{2}$ Department of Electronics and Communication Engineering, Gogte Institute of Technology, India
}

\begin{abstract}
The key objective of super-resolution imaging is the process of reconstructing a high resolution image from one or a set of low resolution images, to overcome the ill-posed conditions of the image capturing process to get better visualization. It has found practical applications in many real world problems such as surveillance, medical imaging, text image analysis, biometrics, satellite imaging, to name a few. This has encouraged many researchers to develop a new super resolution algorithm for a particular purpose. The objective of the review paper is to explore the different image super resolution algorithms used to enhance the low resolution images to high resolution images. This survey on super resolution algorithms will help the researchers to comprehend the effectiveness of the super resolution process and will make easy to develop advanced super resolution methods.
\end{abstract}

Keywords:

Super Resolution, PSNR, Frequency Domain, Gaussian Process Regression, Total Variation

\section{INTRODUCTION}

Human Visual System (HVS) accounts $80 \%$ of information acquired by the people in the form of image or video. Huge and rich information are supported by the video and image as they are the main carriers of perceived data. The acquisition of image and video has become very convenient due to the development of smart mobile phone. Most of the videos and images have to be displayed and stored in low resolution because of the limitation of the bandwidth and storage capacity. Based on low resolution images, high resolution display has to be achieved in order to address the demand of low resolution images clearly played back in HD screen. The size and number of the sensors accounts for the resolution of the captured image. To improve resolution, increasing resolution is not a feasible approach by improving sensor resolution. For example, reduce the pixel size by using sensor manufacturing techniques to increase spatial resolution [1]. The amount of light available also decreases as the pixel size decreases. Due to the generation of the shot noise the image quality gets degraded. To enhance the spatial resolution, another approach is by increasing the chip size which as a result leads to increase in capacitance. This approach is not considered to be effective due to larger capacitance causes complications to speed up a charge transfer rate [2]. To overcome the inherit limitations of the optical imaging systems and the sensors, the new approaches in increasing spatial resolution are required. In many commercial applications, the high cost of the precision optics and image sensors play an important factor. There is a natural loss of spatial resolution while in the process of capturing an image using digital image acquisition system. The factors which contribute to this effect are as follows: for optical distortion ; out of focus, motion blur due to limited shutter speed or relative movement between camera and the object, diffraction limits, noise occurring within the sensor or during transmission and insufficient sensor density. Due to blur, aliasing effects and noise the resulting image is usually degraded. The cause of the distortion in the high frequency contents of the image occurs due to aliasing, wherein the image is sampled at low spatial sampling rate. Improvement in resolution by applying the tools from digital signal processing technique has been of a great topic of interest. Reconstructing high spatial resolution image from one or more low resolution images by using signal processing technique is referred to the term as 'super-resolution'. The contributions of our survey paper is to help the researcher to understand the SR concept by understanding the basic SR model, to learn various methods proposed in the frequency and spatial domain, and advantages and disadvantages of various SR approaches. The section 2 discusses the super resolution model; SR methods proposed researchers in frequency and spatial domain approaches are discussed in section 3 and section 4 respectively. Conclusion and future work is discussed in section 5 .

\section{SUPER RESOLUTION MODEL}

Super resolution is technique to reconstruct the high resolution image using low resolution images. The high frequency content lost during the image acquisition process has to be recovered in the super-resolution techniques. From under-sampled low resolution observations the main concern of the super resolution algorithm is to reconstruct high resolution images, it produces high quality images from blurred, noisy and degraded images. The characteristics of the technique overcoming the inherent resolution limitation of low resolution imaging systems underlines and represents the word 'super'. Some of the advantages of super-resolution approach are i) costs less ii) existing low resolution imaging systems can be still utilized without any additional hardware iii) offers flexibility.

The image restoration problem is closely related to super resolution reconstruction problem. The goal of the image restoration is to recover the image from the degradation such as noise and blur. Hence, for image restoration problem, the size of the restored image and the observed image is the same. Whereas it is different in the image super-resolution since there is a dependency on the decimation factor of the super resolved image. It is necessary to have a detailed understanding of how images are captured and the transformation they undergo in order to apply a super-resolution algorithm. Focusing lens, processor chip, optical sensors, electronic circuits and other mechanical subsystems are usually built in the common digital image acquisition systems (video camera/camcorders/digital cameras). The high resolution image goes through a sequence of degradations such as blur, additive noise and down-sampling when capturing an image of a scene using such camera. There is 
a natural loss of spatial resolution due to optical distortions (out of focus, diffraction limit and insufficient sensor density). Due to causes like relative motion between camera and object, optical aberration, limited shutter speed and atmospheric turbulence the observations could be blurred. The images could also be degraded by various types of noise which occurs during transmission or within the sensor. Due to camera motion like zooming, tilting and panning the frames captured using video camera could be rotated and scaled. Thus due to relative motion between the observations, blur may be introduced. Hence, the observed images account to degraded versions of the high resolution images. A mathematical model that represents the image acquisition process has to be formulated in order to analyze the super-resolution reconstruction problem. The original high resolution (HR) image to be observed low resolution (LR) image(s) is related by this model, known as forward model or observation. The important role in the success of any super-resolution approach is the correct formulation of the observation models. Translation, blur, aliasing and noise in the formulation are the most commonly used forward models for super resolution reconstruction. The Fig. 1 illustrates the typical super resolution model.

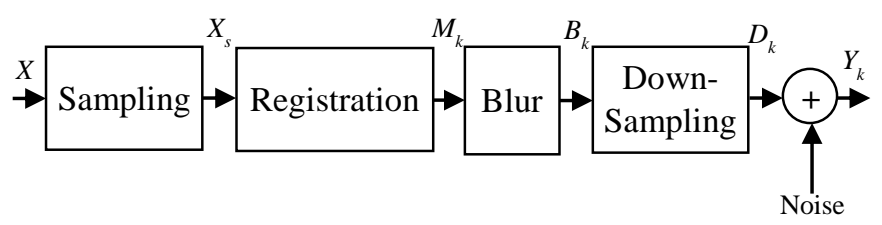

Fig.1. Super Resolution model

Here, $X$ denotes the continuous scene, and $X_{s}$ be the desired HR image sampled above the Nyquist rate from the band-limited continuous scene. The output $Y_{k}$ is the $k^{\text {th }}$ observed LR image from the image sensor. The representation of observation model is:

$$
Y_{k}=D B_{k} M_{k} X+N_{k} \quad k=1,2,3, \ldots, K
$$

where, $D$ is a down-sampling operator, $B_{k}$ contains the blur for the $k^{\text {th }}$ LR image, $M_{k}$ contains the motion information that transforms the $k^{\text {th }}$ LR image onto the HR image grid, and $N_{k}$ is the noise in the $k^{\text {th }}$ LR image. Existing SR methods are classified into frequency domain-based approaches and spatial domain-based approaches.

\section{FREQUENCY DOMAIN-BASED SUPER RESOLUTION}

SR algorithms of frequency domain-based approach first transforms the input LR image(s) to the frequency domain and then estimate the HR image in this domain. Finally, they transform back the reconstructed HR image to the spatial domain. Depending on the transformation employed for transforming the images to the frequency domain, these algorithms are generally divided into two groups: Fourier transform-based and Wavelet transform-based methods, which are explained in the following subsections. Tsai and Huang [3] proposed first frequency domain approach, where the low resolution satellite images are converted in to the discrete Fourier transform (DFT) domain. These images are combined as per the relation between the aliased DFT coefficients of the low-resolution images and that of the unknown high-resolution image. The inverse DFT (IDFT) is applied to the images to transform back to spatial domain to get high resolution images. Vandewalle et al. [4] have proposed a fast superresolution reconstruction based on a non-uniform interpolation using a frequency domain registration. This method has low computation and can be used in the real-time system but the degradation models are limited therefore this algorithm can apply on few applications. The advantages of frequency-domain-based SR approaches are,

- Enhancement of the high-frequency information of the images by extrapolating the high-frequency content available in the low-resolution images and

- Low computational complexity.

The drawback of the frequency-domain based SR methods is insufficient to handle the real-world applications, since they require that there only exists a global displacement between the observed images and the linear space-invariant blur during the image acquisition process i.e. the model is restricted only to global translational motion. Another disadvantage is that, it is difficult to express the prior knowledge which is used to regularize the SR problem. Integration of prior knowledge is necessary to get better results as SR problem is ill-posed

Demirel [5] proposed a super resolution technique in which high frequency sub-band images obtained by discrete wavelet transform (DWT) are interpolated to get high resolution image. The author used stationary wavelet transform (SWT) to enhance the edges. The proposed algorithm enhances Lena, Pepper and Baboon low resolution images having resolution of $128 \times 128$. The achieved PSNR for Lena, Pepper and Baboon is 34.82, 33.06 and 23.87 respectively. The wavelet transform is being applied for addressing the SR problem as it provides efficient multi-scale representation of the image for recovering the high-frequency information [6]. Regularity and structure inherent in interlaced data are utilized to reduce the computation complexity. Separable orthonormal wavelet basis for 2D images is used to obtain a wavelet decomposition using Kronecker products. Khamy et al. [7] [8], proposed wavelet domain based SR method in which registration is performed first and then low-resolution wavelet coefficients are fused a single image. Three low resolution degraded Lena" image of size $128 \times 128$ are used to reconstruct a HR image of size $256 \times 256$. The obtained PSNR is $32.2 \mathrm{~dB}$. A robust wavelet SR approach is proposed by Fermuller [9] [10] which handle registration errors and the blur identification computation. In this wavelet based iterative method is used to reconstruct the high resolution image. Two channel filter bank is used to model the image and based on this limits of high resolution reconstruction is analyzed. Deshpande [11] proposed Discrete Transform Domain based unconstrained image super resolution to super resolve the unconstrained iris images.

\section{SPATIAL DOMAIN-BASED SUPER RESOLUTION}

The spatial domain-based approaches are subdivided in interpolation based approach, reconstruction based approach and learning based approach. Interpolation-based SR image approach: The image interpolation process is the basic operations in image processing. It is a process of resolving the function values at point in between its samples, achieved by fitting a function throughout the discrete samples. Interpolation-based SR methods interpolate 
the HR image from the LR input using a smooth kernel function. Interpolation based SR methods upscale the size of LR by utilizing either parametric or nonparametric methods. To obtain a higher resolution image, this family of methods applies a base function or an interpolation kernel to estimate the pixels in the HR grids. This technique is divided into two groups: non-adaptive and adaptive.

Non-adaptive Interpolation: In this technique, pixels are directly manipulated instead of considering image contents of features. Nearest neighbor [12], Bilinear [13] and Bicubic [14] are examples of this technique. Nearest neighbor interpolation involves the translation of known pixel values. It gives good result when the image has high resolution pixels. In this some information at the edges is lost. In bilinear interpolation the average value of the nearest pixels on either side is used. It is easy to implement. This technique is give better result than nearest neighbor interpolation and take less computation time compare to bicubic interpolation. Bicubic interpolation involves the translation of a weighted average of the nearest pixels. This technique gives better result but take more computational time. When time is not a constraint then this technique give the best result among all the non-adaptive techniques. Spline interpolation involves a special type of piecewise polynomial as the interpolant. Unfortunately, while being of low computational complexity, these approaches suffer from insufficient preservation of spatial details in the image and consequently lower image quality.

Li [15] proposed low computational complex edge-directed interpolation algorithm for natural images. The covariance coefficients from a LR image is estimated first and then use these covariance estimates to adjust the interpolation at a higher resolution based on the geometric duality between the lowresolution covariance and the high-resolution covariance. A hybrid approach of switching between bilinear interpolation and covariance-based adaptive interpolation is proposed to reduce the overall computational complexity. The proposed approach the flower image of resolution of $320 \times 240$ is super resolved to generate image of size $1280 \times 960$. The efficiency of covariancebased adaptation is improved by choosing the window size of 3 . But this approach fails to preserve the isolated dots as these are treated as small edges.

Zhang [16] proposed a new edge-guided nonlinear interpolation technique based on data fusion and directional filter to preserve edge sharpness and reduce ringing artifacts. Two sets of observations are defined in two orthogonal directions to interpolate the pixels. These directional estimates, modeled as different noisy measurements of the missing pixel are fused by the linear minimum mean square-error estimation (LMMSE) technique into a more robust estimate, using the statistics of the two observation sets. The author also proposed a simplified version of the LMMSE-based interpolation algorithm which reduces computational complexity. The proposed method is tested on Lena, Pepper, Butterfly, Cloth and Hole images. The PSNR obtained for these images is: 29.28, 29.28, 28.11, 29.30 and 25.18 respectively which is more than $2 \mathrm{~dB}$ obtained using conventional methods. The author Li (2008) [17] proposed edge-directed interpolation method based on Markov random field (MRF) model which produces smoothness along edges and sharpness across edges. The statistical based approaches are used to implicitly estimate the directions of edges which are indicated by length-16 weighting vectors.

The computational complexity of MRF is reduced by using single-pass implementation. The proposed method is tested for $3 \times 3,5 \times 5,7 \times 7$ and $9 \times 9$ neighborhood structure size on foreman image. The obtained PSNR value for $3 \times 3,5 \times 5,7 \times 7$ and $9 \times 9$ is $34.03,34.25,34.37$ and 34.28 respectively. The computation in seconds for $3 \times 3,5 \times 5,7 \times 7$ and $9 \times 9$ is $89.63,205.42,535.32$ and 811.55

Deshpande [18] [19] proposed iterated back projection and enhanced iterated back projection method to super resolute the long range captured iris polar images. However, interpolationbased SR methods perform well in smooth (low-frequency) areas but poorly in edge (high-frequency) areas in that they are prone to blurring and jaggy artifacts along edges. As a consequence, the SR capability of interpolation-based SR methods is limited. Reconstruction-based SR: The reconstruction-based methods use smoothness prior's knowledge of successive LR image frames of the same scene and solve an ill-posed inverse problem [20] of upsampling, deblurring and denoising for high quality image.

Reconstruction-based algorithms can be classified in two categories: deterministic approach and stochastic approach. Deterministic approach encodes knowledge of what the high resolution image should look like as priors and regularizes the solution using constrained least squares method. The common approach is to impose a smoothness prior via regularization on top of a least-squares optimization. The presence of the regularization term guarantees a convex and differentiable optimization function. Thus, a unique optimal solution can be computed using a number of standard methods like gradient descent. The results of this approach are certainly an improvement over the lowresolution image, but enforcing smoothness is not always the best option, especially if other priors can be formulated that preserve high-frequency details better. Stochastic methods which treat SR reconstruction as a statistical estimation problem have rapidly gained prominence since they provide a powerful theoretical framework for the inclusion of a-priori constraints necessary for satisfactory solution of the ill-posed SR inverse problem. The statistical techniques explicitly handle prior information and noise. Inclusion of prior knowledge is usually more natural using a stochastic approach. The stochastic SR reconstruction using Bayesian approach provides a flexible and convenient way to model a priori knowledge about the final solution. This method can be applied when a posteriori probability density function of the original image can be estimated. The Maximum a posteriori (MAP) approach to estimating the super-resolution seeks the estimate for which the a posteriori probability is a maximum. It is common to utilize Markov random field (MRF) image models as the prior term. Under typical assumptions of Gaussian noise the prior may be chosen to ensure a convex optimization enabling the use of descent optimization procedures.

One advantage of this methods is that it can be easily integrated with other common image processing tasks, such as denoising, de-convolution, enhancement, and so on. An iterative back-projection (IBP) algorithm is proposed by Irani and Peleg [21], where the error between the observed low-resolution images and the simulated low resolution images is used iteratively to estimate the high resolution image. Due to ill-posed nature of SR problem, this approach might not produce unique solution. 
Joshi [22] proposed a SR method to recover the superresolution intensity field from a sequence of zoomed observations. The resolution of the entire scene is obtained at the resolution of the most zoomed observed image which consists of only a small portion of the actual scene. The high resolution image can be modeled as an MRF or as an SAR one and the model parameters were estimated from the most zoomed observation. Subsequently, a MAP estimate is used to restore the superresolved image for the MRF model and a suitable regularization scheme is employed for the SAR model. Marquina [23] proposed a new time dependent convolution model for super-resolution based on a constrained variational model that uses the total variation of the signal as a regularizing functional. The model uses a dataset of low resolution images and incorporates a downsampling operator to relate the high resolution scale to the low resolution one. Lijun [24] proposed wavelet-domain Hidden Markov Tree (HMT) model and Morphological Component Analysis (MCA) model based super resolution algorithm. The author first decomposes texture of image and smooth element, then enhances the smooth part with interpolation. The proposed method is tested on Lena, Bridge and Baboon of resolution $512 \times 512$ pixels. The achieved SSIM is 0.98240 .9598 and 0.9269 , respectively.

$\mathrm{Xu}$ [25] proposes local fractal analysis based single image super resolution algorithm. The image gradient is modeled as an estimate of set of fractals and based on invariance of fractal dimension the image is sharpened. The pixels of a natural image are treated as a fractal set and image gradient is measure of the fractal set. The regularization term is calculated using scale invariance of fractal dimension. This method is tested on Child, Koala and Kodak test images and obtained RMSE 22.14, 12.94 and 17.38 respectively. In this paper, the image gradient was used as a sole measure of fractal, which can be insufficient for some complicated image contents, the ability to preserve edge information has made the Total Variation (TV) model popular among the prior models. Michael [26] proposed the total variation (TV) regularization based super resolution model to deal with the intractable problems like noise, artifacts, motion errors, etc. The author used the fixed-point iteration method and preconditioning techniques to efficiently solve the associated nonlinear EulerLagrange equations of the corresponding variational problem in SR. The algorithm is analyzed by varying the regularization parameter on "Foreman" video sequence of resolution $352 \times 288$ and "Bulletin" video sequence of resolution $640 \times 480$. The author achieved $37.663 \mathrm{~dB}$ at regularization parameter of 0.512 .

Qiang [27] proposed SR algorithm based on a spatially weighted TV. The information distributed on different regions of image, is added to confine the SR method. This method reduces the artifacts produced in fat regions of the image and preserves the edge information. The approach is tested on aerial image of size $200 \times 200$, spot- 5 images of size $256 \times 256$ and cameraman images of size $200 \times 200$ images. The achieved PSNR value for these images is $33.413,32.636$ and 28.65210 respectively. Ren [28] proposed fractional order total variation (TV) regularization for super-resolution to maintain the texture information. This regularization, image fidelity and TV regularization are included into variational formulation to preserve the discontinuities and image structures. The proposed approach suppresses blocking artifacts. This approach is tested on Lena, Pepper, Parrot and Girl images. The obtained SSIM is $0.8188,0.8061,0.8258$ and 0.7305 respectively.

Deshpande [29] [30] [31] proposed a Gaussian process regression (GPR) and total variation based framework to superresolve long range captured iris image. Diamond search algorithm is used to calculate the motion vectors which are used in total variation. In GPR linear kernel co-variance function is used to super resolve the images. Further, the super resolved images are used to recognize the person.

Jieping [32] proposed a super-resolution (SR) technique to reconstruct a higher-resolution image from multiple lowresolution (LR) satellite images. The method first performs image registration in both the spatial and range domains. Then the point spread function (PSF) of LR images is parameterized by a Gaussian function and estimated by a blind de-convolution algorithm based on the maximum a posteriori (MAP). Finally, the high-resolution (HR) image is reconstructed by a MAP-based SR algorithm. The MAP cost function includes a data fidelity term and a regularized term. The data fidelity term is in the L2 norm, and the regularized term employs the Huber-Markov prior which can reduce the noise and artifacts while preserving the image edges.

Shi [33] proposed a novel local and nonlocal total variation combination method for image restoration in wireless sensor networks (WSN). The degraded image is preprocessed by an image smoothing scheme to divide the image into two regions. One contains edges and flat regions by the local TV term. The other is rich in image details and regularized by the nonlocal TV term. Then, the alternating direction method of multipliers (ADMM) algorithm is adopted to optimize the complex object function, and two key parameters are discussed for better performance. Liu [34] proposed a total variation (TV) regularization approach for the reconstruction of super-resolution synthetic aperture radar (SAR) image based on gradient profile prior or other texture image prior in the maximum a posteriori framework. The author also designs a novel super-resolution reconstruction algorithm via split Bregman iteration with the known degradation matrix, thereby enhancing the resolution of the SAR image. The parameter adaptation of the TV regularization is performed based on the high-resolution (HR) SAR image at each step. Several evaluation indices are tested on SAR images for objective assessment of the performance of SAR image super-resolution reconstruction. This computationally efficient algorithm is robust to noise in SAR scenes in HR image estimation. Experimental results show that the proposed split Bregman super-resolution approach can effectively avoid the speckle noise generated due to some strange textures and has good effect of noise suppression, while effectively maintaining the SAR image content, the structure of the SAR image is more apparent. Additionally, the experimental results on real SAR scenes also demonstrate the effectiveness of the proposed algorithm and demonstrate its superiority to other superresolution algorithms.

Because the richness of real-world images is difficult to capture analytically, researchers are exploring a learning-based approach for super-resolving images. This method consists of two phases:

- Training: Image patches dictionary is created. 
- Super-resolution: Up-samples the input image patches using the dictionary.

Two types of dictionaries can be created, external and internal dictionary. The external directory which can be built in advanced using a set of external training images and internal dictionary built without using other images. The internal dictionary uses the property of self-similarity, in which structure of image repeats within and across image of different scales. Learning-based SR approach uses function dependent priors to gather the unknown high resolution image. The input LR image is divided in overlapping or non-overlapping patches. Then the best match is selected from the training database. The output HR image is reconstructed using corresponding HR patches. A method of learning based image super resolution is presented in Wang [35]. It combines both the SR reconstruction constraint and the patch based image co-occurrence constraint in a consistent probabilistic framework. The main strength of the proposed method is its ability to estimate the PSF parameter in the SR process and the SR result is better than existing methods. The proposed probabilistic framework is in fact a combination of the global parametric model and the learned local non-parametric model, and the author believes that it has the potential to solve other type of low level vision problems. Huang [36] proposed Circular Symmetric Filter based SR in which iris feature information of different bands prior relation is predicted and integrated learnt prior into iris enhancement process. The images are divided in $7 \times 7$ patch size and overlapping of 2 pixels used. The proposed approach is tested on CASIA database having 1088 images of 134 objects. 37 classes of images are used for training purpose and 97 classes are used for testing purpose. The achieved recognition rate is $98.86 \%$. This approach is not effective for real LR images.

Chakrabarti [37] proposed a kernel principal component analysis (PCA) based prior model to super resolve the face images. They prior probability is used to regularize the solution based on the energy present in the outside the area of principal components identified in an advanced-dimensional feature space. The proposed approach is tested FERET dataset consisting of 450 of 350 individuals and achieved MSE 98.6.

An example-based approach for video super-resolution is proposed by Brandi et al. (2008) [38]. The high-frequency information of an interpolated block is restored by searching for a similar block in a database and adding the high frequency of the chosen block are added to the interpolated image. The performance is analyzed by testing on Foreman, Mobile and Coast Guard CIF video sequences and achieved PSNR of 32, 23 and 28.5dB. Shin [39] proposed a multiple MLPs (multi-layer perceptrons) based super resolution technique for iris recognition. This method reconstructs high resolution image based on the output values of the three MLPs. The back propagation algorithm is used to train the MLPs. The performance of the proposed approach is tested on CASIA iris database which consists of 2651 images of resolution $320 \times 280$ captured from 396 objects. The MLPs are trained using 12 images from 12 classes. The achieved EER for the resolution of $106 \times 93$ and $80 \times 70$ is $1.018 \%$ and $1.392 \%$.

Wang [40] proposed image super resolution technique based on learning the sparse connection among input image patches and the example patches. The author improved sparse-coding algorithm to find sparse association among image patches. Two training images Lena and Peppers are used to extract the patches centered at edge pixels as pair of patch examples. The edges are detected using Canny edge detector. This generates 6718 and 5719 examples. Baboon, Barbara and Bridge benchmark images are used to evaluate the performance of the proposed approach. For 'lena256' training image, the achieved PSNR in dB is 19.20, 25.82 and 23.82 respectively. For 'lena256' training image, the achieved PSNR in $\mathrm{dB}$ is $19.22,25.52$ and 23.76 respectively.

Tai [41] proposed a super resolution method which is combination of edge-directed SR and learning based SR. This approach uses only a single exemplar image. For large magnification, the better quality image is obtained which is not possible for learning and edge-directed based approaches. This approach is fully dependent on the supplied example image. It produces a sharper image when exemplar image is similar to the ground truth image and it produces different results for different exemplar image.

Zhang [42] proposed a single image SR method by exploiting similarity redundancy across different scales and within the same scale in a given LR image itself. The redundancy across different scales was exploited to achieve example learning-based detail synthesis, where the Neighbor Embedding (NE)-based algorithm was used to estimate the mapping relationship between the LR and HR image pairs. Redundancy within the same scale was utilized to develop a reconstruction-based method, in which the NLM was applied to formulate a nonlocal regularity for reducing the unpleasing artifacts introduced by the NE-based synthesis. To fully exploit similar structures across various scales and to enable more reliable SR results, the author adopted a gradual magnified scheme to magnify the LR input image to desired size step by step.

Due to spontaneous probability interpretation and nonlinear mapping capability, the Gaussian Process (GP) has attracted more attention in the SR [43]-[45]. GP is also implemented on object categorization [46], human motion [47] [48], stereo segmentation [49], etc.

The Gaussian Process Regression is a probability model based on Bayesian theory. Instead of fitting data through a set of predefined basis functions, the GPR learns the intrinsic relationship among data by Gaussian distribution model. Therefore, the method has no constraint on the intrinsic structures of data and can be applied to various linear and nonlinear regression problems flexibly.

A statistical model known as Gaussian process regression was first applied in the reconstruction of high-resolution images in 2011 and has been shown to be capable of generating an image with sharp edges by extracting the necessary information from a low-resolution image. However, it should be noted that this method only uses the local structural information for each pixel's neighborhood, so it can still generate unexpected details.

He et al. [50] proposed a framework for super resolution and deblurring of low resolution image using GPR. GPR predicts the pixels by its neighbors. This method super resolves the image without using external dataset. This approach uses used squared exponential covariance function and patches are overlapped in this process. The approach is tested on Butterfly, Lena and Chip images with up-sampling factor from 2 to 10 . The images are divided in $20 \times 20$ patches. The super resolved images produce sharp edges with least artifacts at a cost of high computation time. 
Xie [51] proposed super resolution technique based on GPR and K-means clustering algorithm to group the training patch as per the local geometric structure of low resolution patches. In this method square exponential covariance function is used find the similarity between image patches. The proposed approach is tested on Butterfly, Girl, Hat and Plants images. The achieved PSNR is $26.16,33.26,30.28$ and 31.56 in $\mathrm{dB}$. This approach produces more remarkable results with few jaggy effects and less noise artifacts. Yan-Yun Qu [52] proposed an approach for super resolving a single image based on data-driven Gaussian Process regression. This approach establishes a relation between the lowresolution space and the high-resolution space free of a regression function. This approach uses external dataset for training purpose which contains pairs of 20,000 low resolution images. The approach is tested using Lena, Child, Oldman and Sculpture. The achieved PSNR in $\mathrm{dB}$ is 29.8040, 25.20, 27.4873 and 30.0803 respectively. Jianmin [53] proposed two algorithms to support local GPR for super resolution. The first algorithm is data driven GPR to learn a GPR model for every LR patch on an input training dataset. The second algorithm is prototype based GPR to improve the running speed by making models for the prototypes of image patches. 100 natural images of group of buildings, animals, people and plants are used to train the database. This approach is tested on Girl, Butterfly, Bike, Lena and Baby and achieved PSNR is 37.5538, 29.6896, 27.0607, 33.5348 and 35.6189 respectively.

Zhu [54] proposed a single image super resolution based on deformable patches where patches are considered as flexible vector. Author presents the energy function with slow and flexible prior for deformation model. During example-based superresolution, the author develops the deformation similarity based on the minimized energy function for basic patch matching. For robustness, author utilizes multiple deformed patches combination for the final reconstruction. Sparse Coding Dictionary Learning and Beta Process Joint Dictionary Learning databases are used for training purpose. The proposed method is tested on Lena, Zebra, Cameraman, Old man and Child images. The obtained PSNR $(\mathrm{dB})$ for up-sampling factor $=3$ and dictionary size $=30,000$ is $31.7536,27.7907,26.2221,30.6666$ and 30.9467.

Dang [55] proposed learning-based super-resolution method rooted in low dimensional manifold representations of highresolution (HR) image-patch spaces. The author exploited the input image and its different down-sampled scales to extract a set of training sample points using a min-max algorithm. A set of low dimensional tangent spaces is estimated from these samples using the norm graph-based technique to cluster these samples into a set of manifold neighborhoods. The HR image is then reconstructed from these tangent spaces.

$\mathrm{Li}$ et al. [56] proposed algorithm is about one-order faster than the data-driven GPR solution because it makes models for the prototypes of image patches rather than for each image patch. Thus, the local regression efforts are greatly reduced to just finding the nearest prototype for each LR image patch and applying its corresponding pre-computed projective matrix for superresolution prediction. The proposed algorithms have greater robustness and usability as they provide a formularized way to automatically learn the hyper-parameters introduced for optimizing the covariance function, while most of the state-of-theart super-resolution methods could only utilize the parameters in a cross-verification way. Li [57] proposed algorithm which uses the field of expert model as the global constraint of SR reconstruction problem to pre-process the low-resolution image. This algorithm classifies the sample patches composed of pre-processed image and high-resolution image, obtains the sub-dictionaries by training, and adaptively selects the most appropriate subdictionary for reconstruction according to the pyramid histogram of oriented gradients feature of image patches. Furthermore, in order to reduce the computational complexity, our algorithm makes use of edge detection, and only applies SR reconstruction based on sparse representation to the edge patches of the test image. Deshpande [58] proposed convolution neural network based super resolution framework to super-resolve the long range captured iris images. By above survey on super resolution techniques, we have summarized the advantages and disadvantages of SR approaches. The comparison of super resolution techniques is as shown in Table.1.

Table.1. Comparison of Super Resolution Techniques

\begin{tabular}{|c|c|c|c|c|}
\hline Methods & Algorithms & Processing & Advantages & Disadvantages \\
\hline $\begin{array}{c}\text { Interpolation } \\
\text { based method } \\
{[12]-[19]}\end{array}$ & $\begin{array}{c}\text { Nearest } \\
\text { neighbor } \\
\text { interpolation } \\
\text { Bi-cubic } \\
\text { interpolation } \\
\text { Bilinear } \\
\text { interpolation }\end{array}$ & $\begin{array}{l}\text { Estimate new } \\
\text { pixel from } \\
\text { neighbor pixel, } \\
\text { interpolate } \\
\text { missing pixel } \\
\text { value into } \\
\text { image to create } \\
\text { HR image. }\end{array}$ & $\begin{array}{l}\text { Simple and } \\
\text { easy to } \\
\text { implement, } \\
\text { fast } \\
\text { computation }\end{array}$ & $\begin{array}{c}\text { interpolation- } \\
\text { based SR } \\
\text { methods } \\
\text { perform well in } \\
\text { low-frequency } \\
\text { areas but } \\
\text { poorly in edge } \\
\text { (high- } \\
\text { frequency) } \\
\text { areas in that } \\
\text { they are prone } \\
\text { to blurring and } \\
\text { jaggy artifacts } \\
\text { along edges }\end{array}$ \\
\hline $\begin{array}{c}\text { Reconstruction } \\
\text { based method } \\
{[20]-[34]}\end{array}$ & \begin{tabular}{|} 
Maximum a \\
posteriori \\
Markov \\
random \\
field \\
Kernel \\
steering \\
Total \\
Variation
\end{tabular} & $\begin{array}{l}\text { Contains three } \\
\text { steps } \\
\text { - Image } \\
\text { registration } \\
\text { - Image fusion } \\
\text { Post } \\
\text { processing }\end{array}$ & $\begin{array}{l}\text { Image } \\
\text { overlay, } \\
\text { change } \\
\text { detection }\end{array}$ & $\begin{array}{c}\text { Small } \\
\text { discontinuity } \\
\text { and loss of } \\
\text { pixels near the } \\
\text { edges }\end{array}$ \\
\hline $\begin{array}{c}\text { Example based } \\
\text { method [35]- } \\
{[57]}\end{array}$ & $\begin{array}{c}\text { Markov } \\
\text { random } \\
\text { field } \\
\text { Sparse } \\
\text { coding } \\
\text { Gaussian } \\
\text { Process } \\
\text { Regression }\end{array}$ & $\begin{array}{c}\text { Estimates HR } \\
\text { image by } \\
\text { comparing } \\
\text { images patches } \\
\text { in } \\
\text { external/internal } \\
\text { image database. }\end{array}$ & $\begin{array}{c}\text { High } \\
\text { magnification } \\
\text { factor }\end{array}$ & $\begin{array}{c}\text { Requires huge } \\
\text { image } \\
\text { database. } \\
\text { Increase in } \\
\text { execution time } \\
\text { as the image } \\
\text { patches of test } \\
\text { image needs to } \\
\text { be searched in } \\
\text { image database }\end{array}$ \\
\hline
\end{tabular}

\section{CONCLUSION AND FUTURE WORK}

In this article, we anticipated to express the notion and growth of super-resolution (SR) over the past three decades. The development in SR techniques can be divided into three stages. In the first stage, researchers shifted their consideration from the study of frequency domain methods to spatial domain algorithms. Regularized multi-frame SR framework were the main focus in the second stage. The GPR and TV frameworks became the most accepted methods due to its performance. There has been a bottleneck-style problem in the development of multi-frame SR, 
while example-based SR for single images has become a hot issue. However, the performance of these algorithms depends on the reliability of the external database. So what should we do in further studies? More advanced, adaptive, and faster methods with extensive applicability are always desirable. In addition, methods should be closely combined with actual requirements. The speedy development of hardware devices will also bring new challenges to the application of the SR framework. However, the possibility of these methods is still limited by the time consumption, and acceleration strategies are essential for largescale applications. In future, Deep learning methods based super resolution approaches will be discussed along with the applications of these approaches.

\section{REFERENCES}

[1] Subhasis Chaudhuri, "Super Resolution Imaging", Kluwer Academic Publishers, 2000.

[2] Sung Cheol Park, Min Kyu Park and Moon Gi Kang, "Super-Resolution Image Reconstruction: A Technical Overview”, IEEE Signal Processing Magazine, Vol. 20, No. 3, pp. 21-36, 2003.

[3] R.Y. Tsai and T.S. Huang, "Multiframe Image Restoration and Registration", Advances in Computer Vision and Image Processing, Vol. 1, pp. 317-339, 1984.

[4] P. Vandewalle, "A Frequency Domain Approach to SuperResolution Imaging from Aliased Low Resolution Images", Master Thesis, Department of Electrical Engineering and Computer Science, University of California, 2004.

[5] Hasan Demirel and Gholamreza Anbarjafari, "Image Resolution Enhancement by using Discrete and Stationary Wavelet Decomposition", IEEE Transactions on Image Processing, Vol. 20, No. 5, pp. 1458-1460, 2011.

[6] N. Nguyen and P. Milanfar, "A Wavelet-based Interpolation-Restoration Method for Super Resolution", Circuits Systems and Signal Processing, Vol. 19, No. 4, pp. 321-338, 2000.

[7] S.E. Ei-Khamy, M.M. Hadhoud, M.I. Dessouky, B.M. Salam and F.E. Abd El-Samie, "Regularized SuperResolution Reconstruction of Images using Wavelet Fusion", Optical Engineering, Vol. 44, No. 9, pp. 1-10, 2005.

[8] S.E. Ei-Khamy, M.M. Hadhoud, M.I. Dessouky, B.M. Salam and F.E. Abd El-Samie, "Wavelet Fusion: a Tool to Break the Limits on LMMSE Image Super-Resolution", International Journal of Wavelet Multi Resolution, Vol. 4, No. 1, pp. 105-118, 2006.

[9] H. Ji and C. Fermuller, "Wavelet-based Super-Resolution Reconstruction: Theory and Algorithm", Proceedings of European Conference on Computer Vision, pp. 262-268, 2006.

[10] H. Ji and C. Fermuller, "Robust Wavelet-based SuperResolution Reconstruction: Theory and Algorithm", IEEE Transactions on Pattern Analysis and Machine Intelligence, Vol. 31, No. 4, pp. 649-660, 2009.

[11] Anand Deshpande and Prashant Patavardhan, "Unconstrained Iris Image Super Resolution in Transform Domain", Proceedings of International Conference on Networking Communication and Data Knowledge Engineering, pp. 173-180, 2017.
[12] R. Franke, "Scattered Data Interpolation: Tests of Some Methods", Mathematics of Computation, Vol. 38, No. 157, pp. 181-200, 1982.

[13] J. Allebach and P.W. Wong, "Edge-Directed Interpolation", Proceedings of IEEE International Conference on Image Processing, pp. 707-710, 1996.

[14] F.N. Fritsch and R.E. Carlson, "Monotone Piecewise cubic interpolation", SIAM Journal on Numerical Analysis, Vol. 17, No. 2, pp. 238-246, 1980.

[15] Xin Li and Michael T. Orchard, "Newedge-Directed Interpolation", IEEE Transactions on Image Processing, Vol. 10, No. 10, pp. 152-1527, 2001.

[16] D. Zhang and Xiaolin Wu, "An Edge-Guided Image Interpolation Algorithm Via Directional Filtering and Data Fusion", IEEE Transactions on Image Processing, Vol. 15, No. 8, pp. 2226-2238, 2006.

[17] X. Li and T.Q. Nguyen, "Markov Random Field Modelbased Edge Directed Image Interpolation", IEEE Transactions on Image Processing, Vol. 7, No. 7, pp. 11211128, 2008.

[18] Anand Deshpande, Prashant Patavardhan and D.H. Rao, "Iterated Back Projection Based Super-Resolution for Iris Feature Extraction”, Procedia Computer Science, Vol. 48, pp. 269-275, 2015.

[19] Anand Deshpande and Prashant Patavardhan, "Single Frame Super Resolution of Non-Cooperative Iris Images", ICTACT Journal on Image and Video Processing, Vol. 7, No. 2, pp. 1362-1365, 2016.

[20] M. Bertero and P. Boccacci, "Introduction to Inverse Problems in Imaging", $1^{\text {st }}$ Edition, Routledge, 1998.

[21] M. Irani and S. Peleg, "Improving Resolution by Image Registration", CVGIP: Graphical Models and Image Processing, Vol. 53, No. 3, pp. 231-239, 1991.

[22] Manjunath V. Joshi, Subhasis Chaudhuri and Rajkiran Panuganti, "A Learning-Based Method for Image SuperResolution from Zoomed Observations", IEEE Transactions on Systems, Man, and Cybernetic-Part B: Cybernetics, Vol. 35, No. 3, pp. 441-456, 2005.

[23] Antonio Marquina and Stanley J. Osher, "Image SuperResolution by TV- Regularization and Bregman Iteration", Journal of Scientific Computing, Vol. 37, No. 3, pp. 367$382,2008$.

[24] Shen Lijun, Xiao Zhi Yun and Han Hua, "Image SuperResolution based on MCA and Wavelet Domain HMT", Proceedings of IEEE International Forum on Information Technology and Applications, pp. 1-8, 2010.

[25] H. Xu, G. Zhai and X. Yang, "Single Image SuperResolution with Detail Enhancement based on Local Fractal Analysis of Gradient", IEEE Transactions on Circuits and Systems for Video Technology, Vol. 23, No. 10, pp. 17401754, 2013.

[26] Michael K. Ng, Huanfeng Shen, Edmund Y. Lam and Liangpei Zhang, "A Total Variation Regularization Based Super-Resolution Reconstruction Algorithm for Digital Video", EURASIP Journal on Advances in Signal Processing, Vol. 4, No. 1, pp. 103-112, 2007.

[27] Qiangqiang Yuan, Liangpei Zhang and Huanfeng Shen, "Multiframe Super-Resolution Employing a Spatially Weighted Total Variation Model", IEEE Transactions on 
Circuits and Systems for Video Technology, Vol. 22, No. 3, pp. 561-574, 2012.

[28] Zemin Ren, Chuanjiang He and Qifeng Zhang, "Fractional Order Total Variation Regularization for Image SuperResolution", Journal Signal Processing, Vol. 93, No. 9, pp. 2408-2421, 2013.

[29] Anand Deshpande and Prashant Patavardhan, "Gaussian Process Regression Based Iris Polar Image Super Resolution", Proceedings of IEEE International Conference on Applied and Theoretical Computing and Communication Technology, pp. 1123-1129, 2016.

[30] Anand Deshpande and Prashant Patavardhan, "Super Resolution of Long Range Captured Multiframe Iris Polar Images”, IET Biometrics, Vol. 6, No. 5, pp. 360-368, 2017.

[31] Anand Deshpande and Prashant Patavardhan, "Multiframe Super-Resolution for Long Range Captured Iris Polar Image", IET Biometrics, Vol. 6, No. 2, pp. 108-116, 2017.

[32] Jieping $\mathrm{Xu}$, Yonghui Liang, Jin Liu and Zongfu Huang, "Multi-Frame Super-Resolution of Gaofen-4 Remote Sensing Images", Sensors, Vol. 17, No. 9, pp. 23-35, 2017.

[33] Mingzhu Shi and Liang Feng, "A Novel Local and Nonlocal Total Variation Combination Method for Image Restoration in Wireless Sensor Networks", EURASIP Journal on Wireless Communications and Networking, Vol. 14, No. 2, pp. 113-118, 2017.

[34] L. Liu, W. Huang and C. Wang, "Texture Image Prior for SAR Image Super Resolution based on Total Variation Regularization using Split Bregman Iteration", International Journal of Remote Sensing, Vol. 38, No. 2, pp. 212-219, 2017.

[35] Q. Wang, X. Tang and H. Shum, "Patch based Blind Image Super-Resolution", Proceedings of IEEE Conference on Computer Vision, pp. 709-716, 2005.

[36] T. Huang, "Earning based Resolution Enhancement of Iris Images", Proceedings of British Machine Vision Conference, pp. 1-6, 2003.

[37] A. Chakrabarti, A.N. Rajagopalan and R. Chellappa, "Super-Resolution of Face Images using Kernel PCA-based Prior", IEEE Transactions on Multimedia, Vol. 9, No. 4, pp. 888-892, 2007.

[38] F. Brandi, R. De Queiroz and D. Mukherjee, "Super Resolution of Video using Key Frames", Proceedings of IEEE International Symposium on Circuits and Systems, pp. 1608-1611, 2008.

[39] K. Shin, "Super-Resolution Method based on Multiple Multi-Layer Perceptrons for Iris Recognition", Proceedings of $4^{\text {th }}$ International Conference on Ubiquitous Information Technologies Applications, pp. 661-668, 2009.

[40] J. Wang, S. Zhu and Y. Gong, "Resolution Enhancement based on Learning the Sparse Association of Image Patches", Pattern Recognition Letters, Vol. 31, pp. 1-10, 2010.

[41] Y.-W. Tai, S. Liu, M.S. Brown and S. Lin, "Super Resolution using Edge Prior and Single Image Detail
Synthesis", Proceedings of IEEE Conference on Computer Vision and Pattern Recognition, pp. 23-29, 2010.

[42] K. Zhang, "Single Image Super-Resolution with Multi-Scale Similarity Learning", IEEE Transactions on Neural Network Learning System, Vol. 24, No. 10, pp. 1648-1659, 2013.

[43] C.E. Rasmussen and C.K.I. Williams, "Gaussian Processes for Machine Learning", MIT Press, 2006.

[44] C.E. Rasmussen and H. Nickisch, "Gaussian Processes for Machine Learning (GPML) Toolbox”, Journal of Machine Learning Research, Vol. 11, pp. 3011-3015, 2010.

[45] K. Ashish, G. Kristan, U. Raphel, D. Trevor, "Gaussian Process for Object Categorization”, International Journal Computer Vision, Vol. 88, No. 2, pp. 169-188, 2010.

[46] R. Urtasun, D.J. Fleet, A. Hertzman and P. Fua, "Priors for People Tracking from Small Training Sets", Proceedings of $10^{\text {th }}$ IEEE International Conference on Computer Vision, pp. 403-410, 2005.

[47] R. Urtasun, D.J. Fleet, A. Hertzman and P. Fua, “3D People Tracking with Gaussian Process Dynamical Models", Proceedings of IEEE International Conference on Computer Vision and Pattern Recognition, pp. 17-22, 2006.

[48] O. Williams, "A Switched Gaussian Process for Estimating Disparity and Segmentation in Binocular Stereo", Proceedings of IEEE International Conference on Advances in Neural Information Processing Systems, pp. 1497-1504, 2006.

[49] H. He and W.C. Siu, "Single Image Super-Resolution using Gaussian Process Regression", Proceedings of IEEE conference proceedings on Pattern Recognition, pp. 449456, 2011.

[50] Fang Xie, Cheng Deng and Jie Xu, "Image Super Resolution using Gaussian Process Regression with Patch Clustering", Proceedings of $5^{\text {th }}$ International Conference on Internet Multimedia Computing and Service, pp. 109-112, 2013.

[51] J. Li, Y. Qu, C. Li, Y. Xie, Y. Wu and J. Fan, "Learning Local Gaussian process Regression for Image SuperResolution", Neurocomputing, Vol. 154, pp. 284-295, 2015.

[52] Yu Zhu, Yanning Zhang and Alan L. Yuille, "Single Image Super-Resolution using Deformable Patches", Proceedings of International Conference on Computer Vision and Pattern Recognition, pp. 166-175, 2014.

[53] Chinh Dang, Mohammad Aghagolzadeh and Hayder Radha, "Image Super-Resolution via Local Self-Learning Manifold Approximation", IEEE Signal Processing Letters, Vol. 21, No. 10, pp. 1123-1138, 2014.

[54] X. Li, J. Chen, Z. Cui, M. Wu and X. Zhu, "Single Image Super-Resolution Based on Sparse Representation with Adaptive Dictionary Selection", International Journal of Pattern Recognition and Artificial Intelligence, Vol. 30, No. 7, pp. 11-17, 2016.

[55] Anand Deshpande and Prashant Patavardhan, (Eds.), "Super Resolution of Long Range Captured Iris Image Using Deep Convolutional Network", Deep Learning for Image Processing Applications, IOS Press, 2017. 